\title{
Influence of interaction time on the red blood cell (dis)aggregation dynamics in vitro studied by optical tweezers
}

\author{
Ruixue Zhu*, Tatiana Avsievich, Alexey Popov, Igor Meglinski \\ Optoelectronics and Measurement Techniques Unit, Faculty of Information Technology and \\ Electrical Engineering, University of Oulu, P.O. Box 4500, Oulu 90014, Finland
}

\begin{abstract}
Optical tweezers (OT) is a unique Nobel Prize winning technology that has been widely used in studying cell interaction dynamics at a single-cell level with highly accurate manipulating of living cells and the ability to detect ultra-low intercellular forces. The reversible aggregation process of red blood cells (RBCs) that strongly influences the blood rheological properties thus being critical for blood microcirculation has long been research studied. However, in spite of plentiful researches dealing with RBC aggregation behavior based on an average response of a large number of cells, the detailed mechanism and the applicability of the two coexistent yet mutually opposed models to this reversible process require additional information. In this study, a two-channel optical tweezers system is utilized to reveal the influence of the cell interaction time $(0-300 \mathrm{sec})$ on the RBC (dis)aggregation dynamics. The results show that for RBC enforced disaggregation in autologous plasma, the longer the two RBCs adhere to each other, the stronger the intercellular interaction is, and the lower the degree that a certain optical pulling force can separate two cells, whereas no significant effect of cell interaction time on RBC aggregation process was observed. This observation indicates that the RBC aggregation and disaggregation in autologous plasma are governed by different mechanisms and that the hysteresis effect, namely the dependence of the disaggregation force on RBC interaction history, is a significant feature that needs special consideration in researches related to the RBC disaggregation process.
\end{abstract}

Keywords: Optical tweezers, red blood cells, aggregation, disaggregation, interaction time

\section{INTRODUCTION}

Red blood cells (RBCs), or erythrocytes, have an intrinsic tendency to form face-to-face two- or three-dimensional binding structures at stasis or low-shear conditions in presence of fibrous biomolecules (like large plasma proteins) or neutral polymers (like dextran), and such structures can be dispersed by high-shear conditions ${ }^{1}$. Measurements and analysis of RBC interaction in (dis)aggregation process have long been a topic of considerable scientific interest not only because it plays a crucial role in blood microcirculation, but also because the pathological status of majority of diseases, including sickle cell anemia, inflammation and thrombin generation, is closely related to the degree of RBC aggregation $^{2}$. The extent and property of cell aggregation in blood circulation rely on the components of the blood plasma, the intrinsic properties of single RBC, as well as various pathological states ${ }^{3}$. Traditional methods for RBC aggregation analysis, including measuring the extent and rate of aggregates formation by optical transmission or reflection properties of the suspension, are generally based on an average response of a large number of cells; detailed information about individual cell-cell interactions cannot be revealed. During the last few decades, with the creation of optical tweezers (OT) as a significant achievement of laser physics ${ }^{4}$, and with the introduction of OT into biological sciences by Nobel laureate Arthur Ashkin ${ }^{5}$, the investigation of intercellular interactions has been rapidly developed at a single-cell level ${ }^{6-8}$. OT has become a vital and indispensable tool in studying cellular and molecular interactions. In our study, RBC (dis)aggregation properties were investigated separately and the results reveal the influence of the cell interaction time $(0-300 \mathrm{sec})$ on both the aggregation and disaggregation processes. The observation of the dependence of the RBC disaggregation force on the cell adhesion time brings new insights into the understanding of dynamic interaction between RBCs and other regulatory studies on this process.

\footnotetext{
* Correspondence: e-mail: ruixue_zhu@outlook.com Phone: +358 417210252
} 


\section{METHODOLOGY}

\subsection{Materials}

The blood samples used to prepare highly diluted RBC suspensions in platelet-free autologous plasma were donated by a single healthy female donor (age 26) and were used in all experiments to avoid deviations caused by individual differences due to different donors. Whole blood samples obtained by venipuncture at a Nordlab clinic (Oulu, Finland) were centrifugated at 6500 RPM (4732 g) for $10 \mathrm{~min}$ to preliminarily separate plasma from RBCs. The residual platelets were further washed out by a second centrifugation $(6500 \mathrm{RPM}, 10 \mathrm{~min})$ of the obtained plasma to get platelet-free plasma. The RBCs were obtained by washing diluted suspension of fingertip prick blood drops in phosphate buffer saline at the same condition every time before the experiments. Experimental samples were finally obtained by diluting around $1 \mu \mathrm{l}$ RBCs in $100 \mu \mathrm{l}$ platelet-free plasma (final concentration was less than 1\%). About 20-30 $\mu 1$ suspension was injected into the sample chamber made by adhering a cover glass to a microscope slide with double-sided tape (100 $\mu \mathrm{m}$ thick) at two edges. Vaseline was used to seal the chamber at the other two free edges after sample injection to avoid evaporation and airflow influence on the measurements. All experiments were conducted within 3 hours after sample preparation at room temperature $\left(22^{\circ} \mathrm{C}\right)$, and with ethical permission (NO. 11/2019) from Finnish Red Cross.

\subsection{Methods}

Optical tweezers (OT) is the main experimental method. The construction of the two-channel OT system is shown in Figure 1, and the basic requirement for optical trapping is the strong focusing of the laser beam. The trapping phenomenon can be explained by momentum transfers involved in laser-particle (RBC) interactions, and a comprehensive description of the physics of optical trapping can be found in the literature ${ }^{9}$. Two orthogonally polarized beams divided by a polarizing beam splitter (PBS) from a single-mode Nd:YAG laser source $(1064 \mathrm{~nm}, 350 \mathrm{~mW}$, Taiwan) are recombined by the second PBS and simultaneously focused by a large numerical aperture water immersion objective (NA=1.00, 100×, Olympus, USA) to form two independent traps in the focal plane. The positions of the two orthogonally polarized beams are controlled by stirring mirrors 1 and 2 , and the power ratio between two beams are controlled with half-wave plates. Real-time observation and recording system is made up of a CMOS camera (Pixelink PL-B621M, Canada) connected to the computer screen, an infrared (IR)-filter, and a LED illumination. The top-view image of the sample chamber shows a pair of trapped RBCs adhering to each other with a linear overlapping distance $x$, and the corresponding interaction area $S_{i}$ is shown in the side-view image. The total surface area of a single RBC is denoted by $S_{0}$. Force calibration based on force equilibrium between the optical trapping force, which is proportional to the trapping power, and the viscous friction force exerted on a trapped RBC by a counterflow with a known velocity is necessary prior to experiments ${ }^{10}$. In our case, the linear relationship between the optical trapping force and the trapping power was obtained as $F_{\text {trap }}=0.2359 * P-0.6684$. Considering that the attenuation coefficient of the objective is about $46.67 \%$, the effective optical trapping power in each trap was up to $30 \mathrm{~mW}$ in our experiments, and the trapping efficiency $Q$ was calculated to be about $0.0455 \pm 0.0016$.

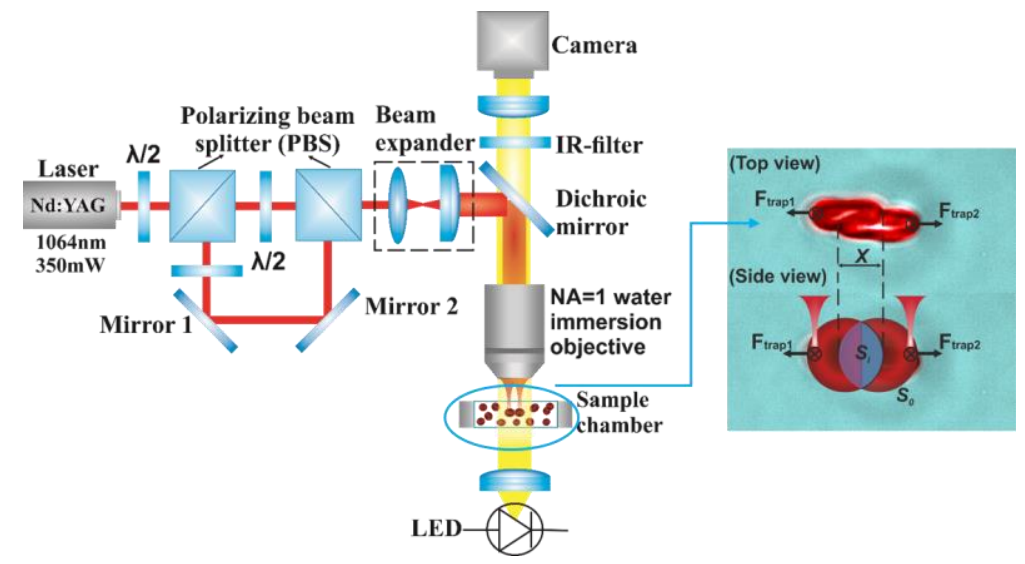

Figure 1. Schematic layout of the two-channel optical tweezers system. Two orthogonally polarized beams separated from one infrared laser source are focused simultaneously by an objective with large numerical aperture to form two independent trapping channels. The beam positions in the focal plane are controlled by stirring mirrors 1 and 2 , and the power ratio 
between the two traps is controlled with half-wave plates. Two trapped RBCs adhering to each other with a stable linear overlapping distance $x$ are shown in the top view image of the sample chamber, and the corresponding interaction area between the two cells can be seen from the side view image.

\section{RESULTS}

The influence of cell interaction time on the adhesion of red blood cells (RBCs) was studied for the aggregation and disaggregation process separately, as the intercellular forces involved in these two processes have significantly different orders of magnitude 7 . There are currently two coexistent yet mutually opposed models for the mechanism of this reversible RBC aggregation process. Among them, the 'depletion layer model', which interprets the RBC clumping as the osmotic pressure caused by a depletion layer of low macromolecule concentration near the interaction surfaces between two RBCs, has been widely used to describe the aggregation process ${ }^{11}$. The 'bridging model' assuming that the two RBCs in contact are connected by 'bridges' formed by physisorption of macromolecules between two cells is widely applied to interpret the intercellular interaction in disaggregation process ${ }^{12}$.

Two separate RBCs resting on the microscope slide were captured and lifted to a height of $40 \mu \mathrm{m}$ by the two trapping channels simultaneously. By controlling the positions of the traps, the RBCs were put into contact and a stable linear overlapping distance was formed as shown in the top view image in Figure 1. For the disaggregation force measurement, the trap 2 was slowly moved away from the trap 1 in an attempt to separate two RBCs while the trapping force/power in each trap was kept still. When the interaction force between the two cells matched and started to be stronger than the trapping force, the cells escaped the trap and started clumping together. The extent to which two RBCs can be separated by a certain optical pulling force is represented by the relative final remaining contact area between the two cells $\left(S_{i} / S_{0}\right)$ : a larger final overlap $\left(S_{i} / S_{0}\right)$ indicates a lower separation degree achieved by the given force. Two optical trapping forces $(9.24 \mathrm{pN}$ and $5.39 \mathrm{pN})$ were adopted in two independent measurement groups for the evaluation of the influence of cell interaction time on the disaggregation process. The initially formed linear overlapping distance $x$ between two RBCs was controlled to be about $80 \%$ in all experiments. The attempt to separate two cells by moving the trap 2 was started after a certain interaction time varied from $0 \mathrm{~s}$ to $300 \mathrm{sec} .48$ pairs of RBCs in total were measured (at least 3 pairs of RBCs for each interaction time). Results obtained by the trapping force of $9.24 \mathrm{pN}$ are shown in Figure 2(a), and a comparison between the results obtained by $5.39 \mathrm{pN}$ and $9.24 \mathrm{pN}$ is shown in Figure 2(b). The photomicrographs showing the moments before the cells escaped from the traps in Figure 2(a) demonstrate the different final degree of separation between two RBCs achieved by a same optical pulling force of $9.24 \mathrm{pN}$ after different cell interaction time. It can be seen that the longer the interaction time, the lower the degree that a certain force can separate two RBCs. This effect is more prominent within the first $60 \mathrm{~s}$ of RBCs interaction, and the cell adhesion becomes more stable after a longer period of contact with each other. In addition, as shown in Figure 2(b), the difference in the separation degree between two RBCs after different interaction time observed in the experimental group with an optical pulling force of $5.39 \mathrm{pN}$ is not significant, especially in comparison with the results of the $9.24 \mathrm{pN}$ group. This indicates that the effect of cell interaction time on the RBC disaggregation process is reliably detectable when the optical pulling force is stronger. Our results are consistent with alternative experimental observation within the first few dozen seconds ${ }^{13}$ and explain the reason for not observing this influence in the case of a small (about $6 \mathrm{pN}$ ) trapping force ${ }^{14}$.

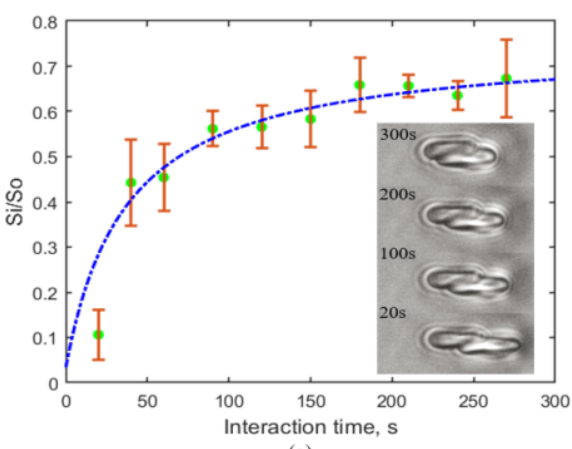

(a)

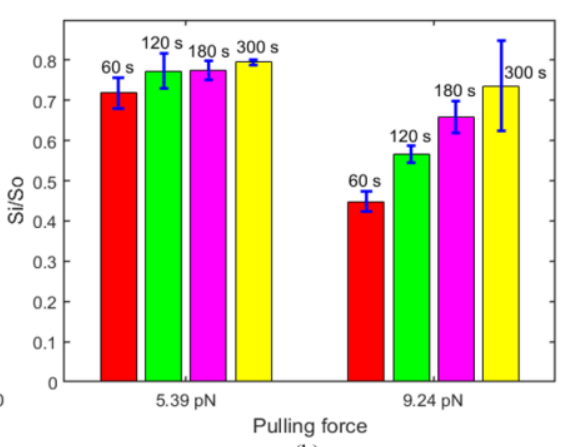

(b)

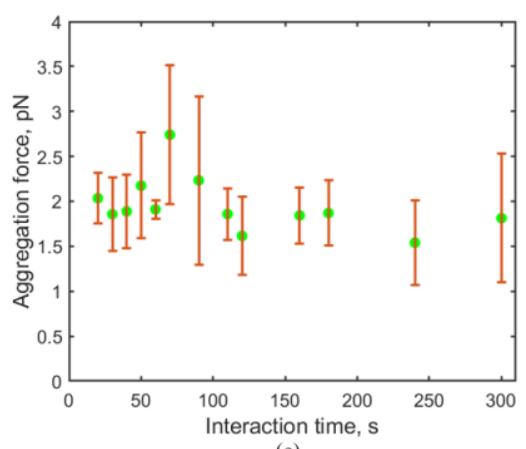

(c)

Figure 2. (a) Time dependence of the final relative interaction area $\left(S_{i} / S_{0}\right)$ achieved by a trapping force of $9.24 \mathrm{pN}$ from an $80 \%$ initial relative overlapping area in the disaggregation process. The green dots show the experimental data with standard 
deviations and the dashed curve denotes a possible trend of interaction time influence. Image sequence illustrates the longer the interaction time, the lower the achievable separation degree between two RBCs by $9.24 \mathrm{pN}$ optical pulling force. (b) Comparison of the results obtained by two different forces $(5.39 \mathrm{pN}$ and $9.24 \mathrm{pN})$, indicating the interaction time effect is more detectable with a larger trapping force. Results of four different interaction times are expressed in different colors. (c) Aggregation forces measured with 39 pairs of RBCs with an initial relative interaction area $\left(\mathrm{Si}_{i} / \mathrm{S}_{0}\right)$ of around $40 \%$ over different interaction times $(0-300 \mathrm{~s})$. No significant influence of interaction time on the aggregation process was observed.

In the aggregation process, the initially formed linear interaction distance $x$ between the two RBCs was kept still (40\%) while the trapping power was slowly decreased. Once the optical trapping force matched and started to be weaker than the cell interaction force generated by this $40 \%$ adhesion, the RBC escaped the trap and clumped together. At that escaping point, the optical trapping force was considered equal to the RBC aggregation force $\left(F_{A}\right)$ upon this contact area. The aggregation forces of 39 pairs of RBCs were measured over different interaction times $(0-300 \mathrm{~s})$ (at least 3 pairs of RBCs for each interaction time). As shown in Figure 2(c), the obtained aggregation force was $1.95 \pm 0.51 \mathrm{pN}$, with no significant effect of interaction time on RBC aggregation process observed in autologous plasma. The large standard deviations that occur at cell contact times of $70 \mathrm{~s}$ and $90 \mathrm{~s}$ are due to measurement variability.

\section{CONCLUSIONS AND ACKNOWLEDGEMENTS}

The effects of cell interaction time up to $300 \mathrm{~s}$ on both the RBC aggregation and disaggregation processes in autologous plasma were measured separately with different measurement methods. No significant influence was observed in the aggregation force measurements. However, the results for the RBC disaggregation measurements indicate that the longer the two RBCs adhere to each other, the more stable the intercellular interaction is, and the lower the degree that a certain optical pulling force can separate two cells. This observation indicates that the RBC aggregation and disaggregation in autologous plasma are governed by different mechanisms, and that the 'bridges' formation between RBCs involved in the 'bridging model' for the disaggregation process has a dependence on the cell contact time. Our study provides new insights into the investigation of the dynamic interaction between RBCs in autologous plasma and points out that the hysteresis effect, namely the dependence of the disaggregation force on the RBCs interaction history, is a significant feature that needs special consideration in researches related to the RBC disaggregation process.

China Scholarship Council (scholarship No. 201706410089, RZ), Tauno Tönning Foundation (project No. 20190104, RZ), EDUFI Fellowship (TM-17-10370, TM-18-10820, TA), Academy of Finland (grant No. 290596, 314369, AP) are acknowledged for financial support.

\section{REFERENCES}

[1] Baskurt, O. K. and Meiselman, H. J., "Erythrocyte aggregation: Basic aspects and clinical importance," Clin. Hemorheol. Microcirc., 53(1-2), 23-37 (2013).

[2] Horne III, M. K., Cullinane, A. M., Merryman, P. K. and Hoddeson, E. K., "The effect of red blood cells on thrombin generation," British journal of haematology, 133(4), 403-408 (2006).

[3] Rampling, M. W., Meiselman, H. J., Neu, B. and Baskurt, O. K., "Influence of cell - specific factors on red blood cell aggregation," Biorheology, 41(2), 91-112 (2004).

[4] Ashkin, A., "Acceleration and Trapping of Particles by Radiation Pressure," Phys. Rev. Lett. 24(4), 156 (1970).

[5] Ashkin, A. and Dziedzic, J. M., "Optical trapping and manipulation of viruses and bacteria," Science, 235(4795), 1517-1520 (1987).

[6] Avsievich, T., Popov, A., Bykov, A. and Meglinski, I., "Mutual interaction of red blood cells assessed by optical tweezers and scanning electron microscopy imaging," Opt. Lett., 43(16), 3921-3924 (2018).

[7] Lee, K., Danilina, A.V., Kinnunen, M., Priezzhev, A.V. and Meglinski, I., "Probing the Red Blood Cells Aggregating Force with Optical Tweezers," IEEE J. Sel. Top. Quantum Electron, 22(3), 7000106 (2016).

[8] Lee, K., Priezzhev, A., Shin, S., Francois, Y. and Meglinski, I., "Characterization of shear stress preventing red blood cells aggregation at the individual cell level: the temperature dependence," Clin. Hemorheol. Microc., 64(4), $853-857$ (2016). 
[9] Nieminen, T. A., Knöner, G., Heckenberg, N. R. and Rubinsztein - Dunlop, H., "Physics of optical tweezers," Methods in cell biology, 82, 207-236 (2007).

[10] Avsievich, T., Popov, A., Bykov, A. and Meglinski, I., "Mutual interaction of red blood cells influenced by nanoparticles," Scientific reports, 9(1), 5147 (2019).

[11] Neu, B. and Meiselman, H. J., "Depletion-mediated red blood cell aggregation in polymer solutions," Biophysical journal, 83(5), 2482-2490 (2002).

[12] Tozeren, A., Sung, K. L. and Chien, S., "Theoretical and experimental studies on cross-bridge migration during cell disaggregation," Biophys. J., 55(3), 479 (1989).

[13] Khokhlova, M., Lyubin, E.V., Zhdanov, A.G., Fedyanin, A.A., Rykova, S.Y. and Sokolova, I.A., "Normal and system lupus erythematosus red blood cell interactions studied by double trap optical tweezers: direct measurements of aggregation forces," J. Biomed. Opt., 17(2), 025001 (2012).

[14] Lee, K., Kinnunen, M., Khokhlova, M. D., Lyubin, E. V., Priezzhev, A. V., Meglinski, I. and Fedyanin, A. A., "Optical tweezers study of red blood cell aggregation and disaggregation in plasma and protein solutions," J. Biomed. Opt., 21(3), 035001 (2016). 